\title{
HPV-Impfung: Impfabstand ausschlaggebend für die Effektivität
}

\author{
Populationsbezogene Untersuchungen zur quadrivalenten HPV-Impfung \\ belegen einen hohen dosisabhängigen Schutz vor Genitalwarzen. Bei \\ Anwendung des 2-Dosen-Impfschemas ist die Wirksamkeit der Impfung \\ abhängig vom Intervall zwischen der ersten und zweiten Dosis.
}

n vielen Ländern werden Impfprogramme gegen humane Papillomviren heute erfolgreich umgesetzt. Es gilt inzwischen als unbestritten, dass die beiden HPVImpfstoffe bei der Verhinderung höhergradiger zervikaler Läsionen hoch effektiv sind. Wichtige Faktoren für den Erfolg eines Impfprogrammes sind nicht nur eine hohe Durchimpfquote, sondern auch die Vervollständigung der Grundimmunisierung. Nach der Zulassung der HPVImpfstoffe erfolgte die Grundimmunisierung mit drei Dosen. Seit 2014 ist auch ein 2-Dosen-Impfschema für Mädchen im Alter von 9-13 Jahren (quadrivalenter Impfstoff) bzw. 9-14 Jahren (bivalenter Impfstoff) basierend auf immunologischen Brückenuntersuchungen zugelassen. Da Genitalwarzen ein hervorragender Surrogatmarker für die klinische Wirksamkeit des quadrivalenten Impfstoffs sind, lässt sich an der Reduktion von Kondylomen die Effektivität des 2-Dosen-Impfschemas abschätzen. Hierzu wurden populationsbezogene Daten zur Inzidenz von Kondylomen bei HPVgeimpften Mädchen der Geburtsjahrgänge 1985-1999 in Dänemark ausgewertet. Von der Gesamtkohorte von 550.690 Mädchen erhielten 361.734 den quadrivalenten Impfstoff, der für das nationale Impfprogramm verwendet wurde. $15,1 \%$ waren nur einmal, 25,9\% zweimal und $58,8 \%$ dreimal geimpft worden. Mädchen, bei denen Genitalwarzen bereits vor der Impfung aufgetreten waren, wurden von der Auswertung ausgeschlossen. Die Inzidenz von Genitalwarzen betrug bei nicht geimpften Mädchen 655,6 pro 100.000 (95\% Konfidenzintervall (KI): 645,0-666,4) im Vergleich zu 333,3 (95\% KI: 301,0-369,1) nach einer Dosis, 146,4 (95\% KI: 129,6-165,2) nach zwei Dosen und 67,2 (95\% KI: 61,0-74,2) nach drei Dosen. Bei der detaillierten Auswertung der Mädchen, die zwei Dosen erhalten hatten, zeigte sich, dass die Inzidenz von
Genitalwarzen mit zunehmendem Abstand zwischen der ersten und zweiten Impfdosis abnahm. Beim Vergleich zum Impfintervall von zwei Monaten zwischen der ersten und zweiten Dosis wurde die Inzidenz bei vier Monaten um 45\% (95\% KI: 20-62), bei fünf Monaten um 55\% (95\% KI: 35-69) bei sechs Monaten um 63\% (95\% KI: 44-75) reduziert. Die Studie zeigte, dass durch ein 3-DosenImpfschema ein besserer Schutz vor Genitalwarzen erreicht wird als durch zwei Dosen. Wird der Abstand zwischen der ersten und zweiten Dosis dagegen auf sechs Monate ausgedehnt, ergibt sich eine Protektionsrate, die in der Größenordnung des 3-Dosen-Impfschemas liegt.

Blomberg $\mathrm{M}$ et al.: Dose-related differences in effectiveness of human papillomavirus vaccination against genital warts: a nationwide study of 550000 young girls. Clin Infect Dis. 2015; 61:676-82

\section{Kommentar}

Diese populationsbezogene Untersuchung zur Effektivität der HPV-Impfung erlaubt mehrere wichtige Folgerungen. Nur durch die Vervollständigung der Grundimmunisierung wird ein wirksamer Schutz gegen HPV induziert. Bei vielen Mädchen/Frauen in Deutschland wurde besonders 2008 und 2009 die Impfserie nicht beendet. Zum anderen decken sich die jetzt berichteten klinischen Effektivitätsdaten mit den immunologischen Untersuchungen zum 2-DosenImpfschema. Für einen hohen Schutz nach zwei Impfdosen korreliert der zunehmende Abstand zwischen der ersten und zweiten Dosis auf sechs Monate mit der Wirksamkeit.

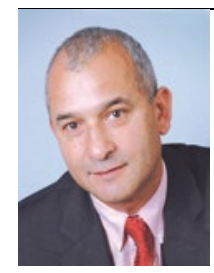

Prof. Dr. med. Tino F. Schwarz Zentrallabor, Stiftung Juliusspital Würzburg

\section{Hier steht eine Anzeige.}

\author{
Springer
}

